\title{
Centrifugal Acceleration in Pulsar Magnetospheres
}

\author{
R. M. C. Thomas \& R. T. Gangadhara \\ Indian Institute of Astrophysics, Bangalore 560034, India
}

\begin{abstract}
We present a relativistic model of pulsar radio emission by plasma accelerated along the rotating magnetic field lines projected on to a $2 \mathrm{D}$ plane perpendicular to the rotation axis. We have derived the expression for the trajectory of a particle, and estimated the spectrum of radio emission by the plasma bunches. We used the parameters given by Peyman \& Gangadhara (2002). The analytical expressions for the Stokes parameters are obtained, and their values compared with the observed profiles. The one sense of circular polarization, observed in many pulsars, can be explained in light of our model.
\end{abstract}

\section{Introduction}

It is important to understand the charged particle dynamics in the pulsar magnetosphere to unravel the radiation mechanism of pulsars. The particles are constrained to move strictly along the field lines, owing to the super-strong magnetic field. The equation of motion for a charged particle moving along the rotating field line is given by Gangadhara (1996). Here we extend this work to obtain an analytical expression for particle trajectory and Stokes parameters. The pulsar rotation effects such as aberration and retardation can create asymmetric pulse profiles

\section{Dynamics of a Charged Particle}

The equation of motion of a charged particle along a rotating magnetic field line is given by (Gangadhara 1996):

$$
\frac{d}{d t}\left(m \frac{d r}{d t}\right)=m \Omega^{* 2} r, \quad \gamma=\left(1-\frac{\dot{r}^{2}}{c^{2}}-\frac{r^{2} \Omega^{* 2}}{c^{2}}\right)^{-1 / 2}
$$

where $m=m_{0} \gamma$ is the relativistic electron mass, $m_{0}$ is the rest mass, $\mathrm{c}$ is the speed of light, $\gamma$ is the Lorentz factor, $\Omega^{*}=\Omega \sqrt{1-b^{2} / r^{2}}$ is the effective angular velocity of the particle, and $b=d_{0} \cos \theta_{0}$. We have solved this equation and obtained the analytical solution:

$$
r=\frac{c \sqrt{1+D^{2}}}{\Omega} c n(\lambda-\Omega t)
$$



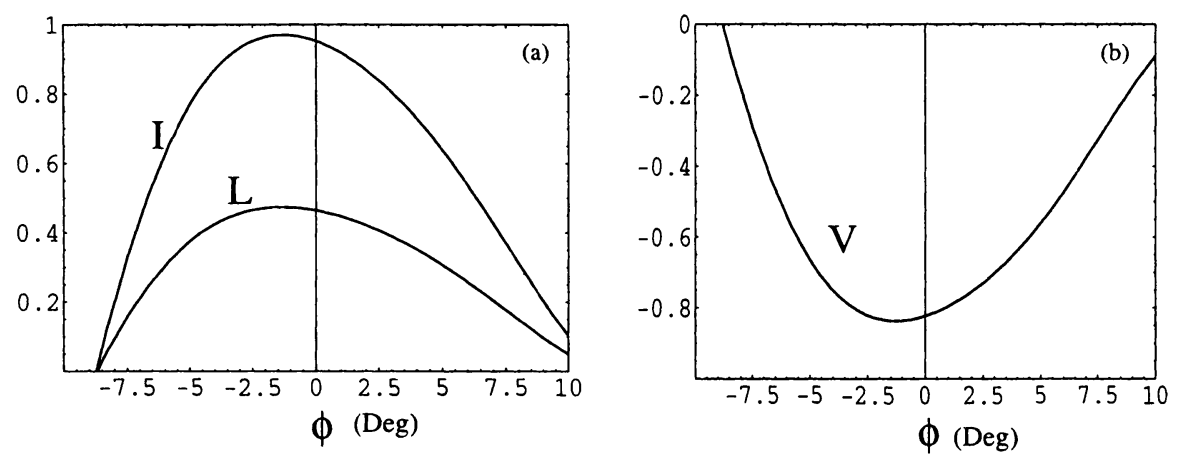

Figure 1. (a) Intensity and linear polarization vs rotation phase, $\phi$, for the radiation emitted by particles accelerated along the rotating magnetic field lines; (b) circular polarization of the emitted radiation.

where $\operatorname{cn}(\lambda-\Omega \mathrm{t})$ is the Jacobian Elliptical cosine function (Abramowitz \& Stegun 1972),

$$
D=\frac{\Omega d_{0} \cos \theta_{0}}{c}, \quad \lambda=\int_{0}^{\phi_{0}} \frac{d \zeta}{\sqrt{1-k^{2} \sin \zeta^{2}}}, \quad \phi_{0}=\arccos \left(\frac{r_{0} \Omega}{c}\right),
$$

$r_{o}$ the initial particle injection point, $d_{o}$ the distance between magnetic pole and rotation axis in the projected $2 \mathrm{D}$ plane, $\theta_{o}$ the initial injection angle with respect to the meridional plane, $\Omega$ the angular velocity of the pulsar, $k$ is an integration constant, $\zeta$ is a dummy variable, and $\theta$ is the angle that the line of sight makes with the 2D plane. Using the expression for $r$, an approximate value for $\rho$, the radius of curvature of the particle in the lab frame, is found to be $\rho \approx \sqrt{1+D^{2}} r_{L} / 2$, where $r_{L}$ is the light cylinder radius.

For a pulsar with period of $1 \mathrm{~s}$, we estimated the components of the electric field of radiation and hence the Stokes parameters. In Figure 1(a), we have plotted the intensity, $I$, linear polarization, $L$,; in Fig. 1(b) circular polarization, $V$, is plotted. Due to aberration-retardation phase shift, the profile is asymmetric about the center, $(\phi=0)$, of the profile. It also shows that leading part of the profile is stronger than the trailing part. Since the line of sight stays above or below the plane of the particle trajectory, circular polarization is produced in one sense, as observed in many pulsars.

\section{References}

Abramowitz, M., \& Stegun, I., A. 1972, A Handbook of Mathematical Functions, (New York: Dover)

Gangadhara, R. T. 1996, A\&A, 314, 853

Peyman, A., \& Gangadhara, R. T. 2002, ApJ, 566, 365 